\title{
Myocardial fibrosis as a early cardiac marker of disease in patients with lamin $\mathrm{A} / \mathrm{C}$ mutations
}

\author{
Andrea Barison ${ }^{1 *}$, Pier G Masci ${ }^{2}$, Marianna Fontana ${ }^{2}$, Matteo Milanesi ${ }^{2}$, Roberta Poletti ${ }^{2}$, Vincenzo Positano ${ }^{2}$, \\ Claudio Passino ${ }^{1}$, Giovanni D Aquaro², Giancarlo Todiere², Michele Emdin², Massimo Lombardi² \\ From 2011 SCMR/Euro CMR Joint Scientific Sessions \\ Nice, France. 3-6 February 2011
}

\section{Objective}

to assess myocardial fibrosis in lamin $\mathrm{A} / \mathrm{C}$ mutations (LM) carriers using constrast-enhanced cardiac magnetic resonance (CMR).

\section{Background}

LM carriers usually develop a dilated cardiomyopathy (DCM) phenotype in association with atrio-ventricular blocks and/or ventricular arrhythmias. However, sudden cardiac death may be the first clinical manifestation. Hence early detection of myocardial abnormalities before overt left ventricular (LV) dysfunction is warranted for a better risk stratification.

\section{Methods}

Seventeen paucisymptomatic LM carries (age $41 \pm 16$ years, 9 male), 14 paucisymptomatic DCM patients (age $59 \pm 11$ years, 8 male, NYHA I-II) with mild LV dysfunction (ejection-fraction 40\%-55\%) and 12 healthy controls (age $46 \pm 10$ years, 9 male) underwent complete clinical, echocardiographic, biohumoral and contrast-enhanced CMR assessment. Cine CMR was used to derive LV volumes, mass and function, and post-contrast CMR to detect late gadolinium enhancement (LGE) as an index of gross fibrosis. Eight LM patients, all DCM patients and all normal controls underwent pre- and post-contrast myocardial T1 mapping with gadolinium partition coefficient calculation, as an index of interstitial fibrosis.

\section{Results}

LM carriers presented a similar LV end-diastolic volume $(85 \pm 17 \mathrm{ml} / \mathrm{m} 2, \mathrm{p}=\mathrm{NS})$ and slightly reduced ejection-fraction $(61 \pm 6 \% \mathrm{p}<0.001)$ than controls $(83 \pm 19 \mathrm{ml} / \mathrm{m} 2$,

'Scuola Superiore Sant'Anna and Fondazione G Monasterio CNR - Regione Toscana, Pisa, Italy

Full list of author information is available at the end of the article
$67 \pm 6 \%$ ), while in DCM patients LV end-diastolic volume $(102 \pm 17 \mathrm{ml} / \mathrm{m} 2)$ and ejection-fraction $(49 \pm 5 \%)$ were worse than both controls and LM carriers $(\mathrm{p}<0.001)$. Moreover, DCM patients presented worse diastolic dysfunction $(9 \pm 3$ vs $7 \pm 1, \mathrm{p}<0.01)$, NT-proBNP plasma levels $(438 \pm 416$ vs $108 \pm 139 \mathrm{ng} / \mathrm{l}, \mathrm{p}<0.01)$ and Doppler-estimated pulmonary pressure ( $33 \pm 7$ vs $30 \pm 5 \mathrm{mmHg}, \mathrm{p}<0.01)$ than LM carriers. Six (43\%) DCM patients presented DE, representing 9 $\pm 3 \%$ of LV mass; similarly, seven (42\%) LM carriers had DE, but with a larger extension $(11 \pm 5 \%$ of LV mass, $\mathrm{p}<0.03)$. In LM carries DE distribution was patchy (2 pts) or midwall (5 pt), similarly to DCM patients (patchy in $1 \mathrm{pt}$, midwall in 4 pts, subendocardial in $1 \mathrm{pt}$ ). GPC reached a steady state 5 minutes after gadolinium injection. In DCM patients GPC was slightly higher than controls $(0.42 \pm 0.05$ vs $0.38 \pm 0.06, \mathrm{p}=0.04)$, while in LM carriers it was much higher $(0.45 \pm 0.07)$ than controls $(\mathrm{p}<0.01)$. Even excluding hyperenhanced myocardium, in LM carriers GPC remained higher than controls, while in DCM patients only slightly higher than controls.

\section{Conclusions}

Myocardial fibrosis occurs early in LM carrier before LV dilatation and dysfunction and may represent an early phenotypic expression of cardiac involvement.

\section{Author details \\ 'Scuola Superiore Sant'Anna and Fondazione G Monasterio CNR - Regione \\ Toscana, Pisa, Italy. ${ }^{2}$ Fondazione G. Monasterio CNR - Regione Toscana, Pisa, Italy.}

Published: 2 February 2011

doi:10.1186/1532-429X-13-S1-076

Cite this article as: Barison et al.: Myocardial fibrosis as a early cardiac

marker of disease in patients with lamin A/C mutations. Journal of

Cardiovascular Magnetic Resonance 2011 13(Suppl 1):076.

\section{Biomed Central}

(c) 2011 Barison et al; licensee BioMed Central Ltd. This is an open access article distributed under the terms of the Creative Commons Attribution License (http://creativecommons.org/licenses/by/2.0), which permits unrestricted use, distribution, and reproduction in any medium, provided the original work is properly cited. 